\title{
Fermilab
}

Managed by Fermi Research Alliance, LLC for the U.S. Department of Energy Office of Science

\section{STATISTICAL ANALYSIS OF THE EIGENMODE SPECTRUM IN THE SRF CAVITIES WITH MECHANICAL IMPERFECTIONS}

A. Lunin, T. Khabiboulline, N. Solyak, A. Sukhanov, V. Yakovlev ICAP'18, Key West, USA 20-24 October, 2018 


\section{Motivation}

HOMSC2014, A. Sukhanov et., all

3.9 $\mathrm{GHz}$ cavity profile deviation*

- SRF cavities are very good resonance systems with multiple eigenmodes (HOMs) with very low losses (high Q-factors)

- Beam of charged particles interacts with HOMs in SRF cavities

- Single bunch interaction

- incoherent losses and wake fields

- CW beam may have beam harmonics close to HOMfrequencies

- resonance excitation of HOMs

- at exact resonance beam power loss may be high

- for monopole modes:

$\left.P_{\text {loss }}=I_{n}^{2} R / Q\right)_{m} Q_{\text {L }}$

- For a single cavity analysis of non-propagating modes is sufficient

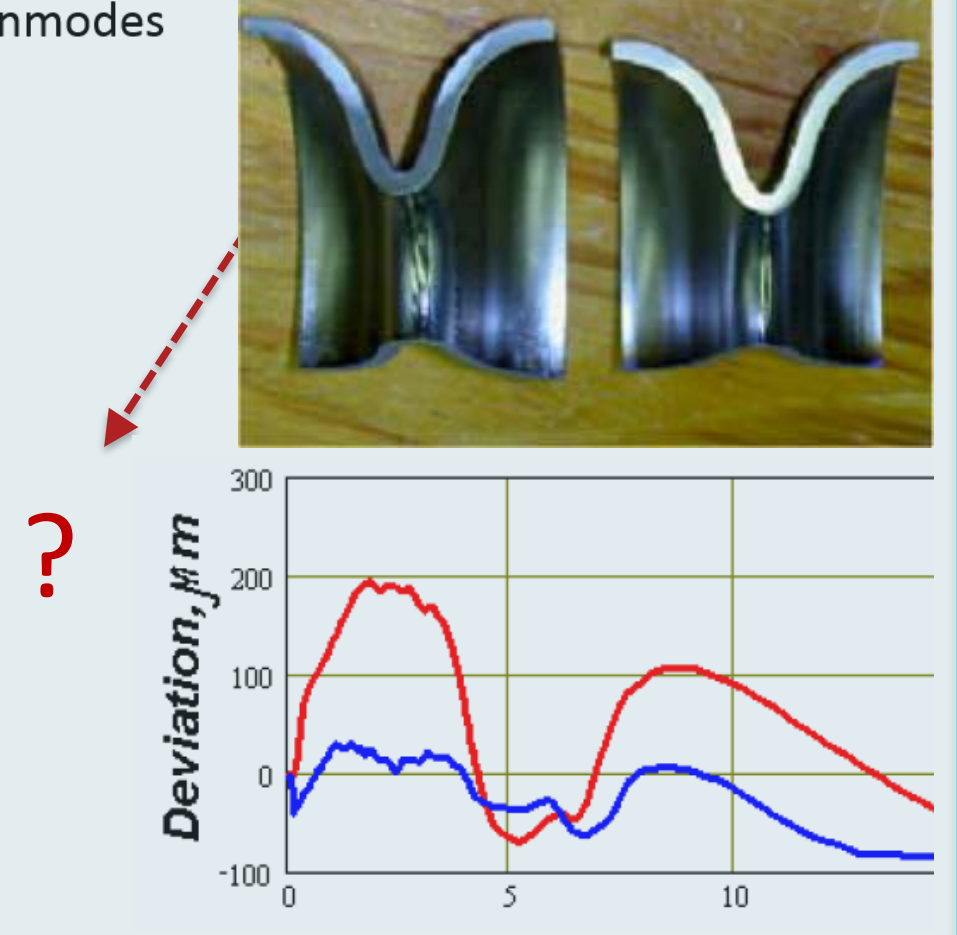

- HOMs parameters deviate from nominal values due to cavity imperfections.

- Coherent HOM excitation is essentially the probabilistic problem!

- Finding HOMs spread is essential for the probability estimation

* N. Solyak et al., TPAB014,, in Proc. PAC 2003 


\section{Coherent HOM Excitation}
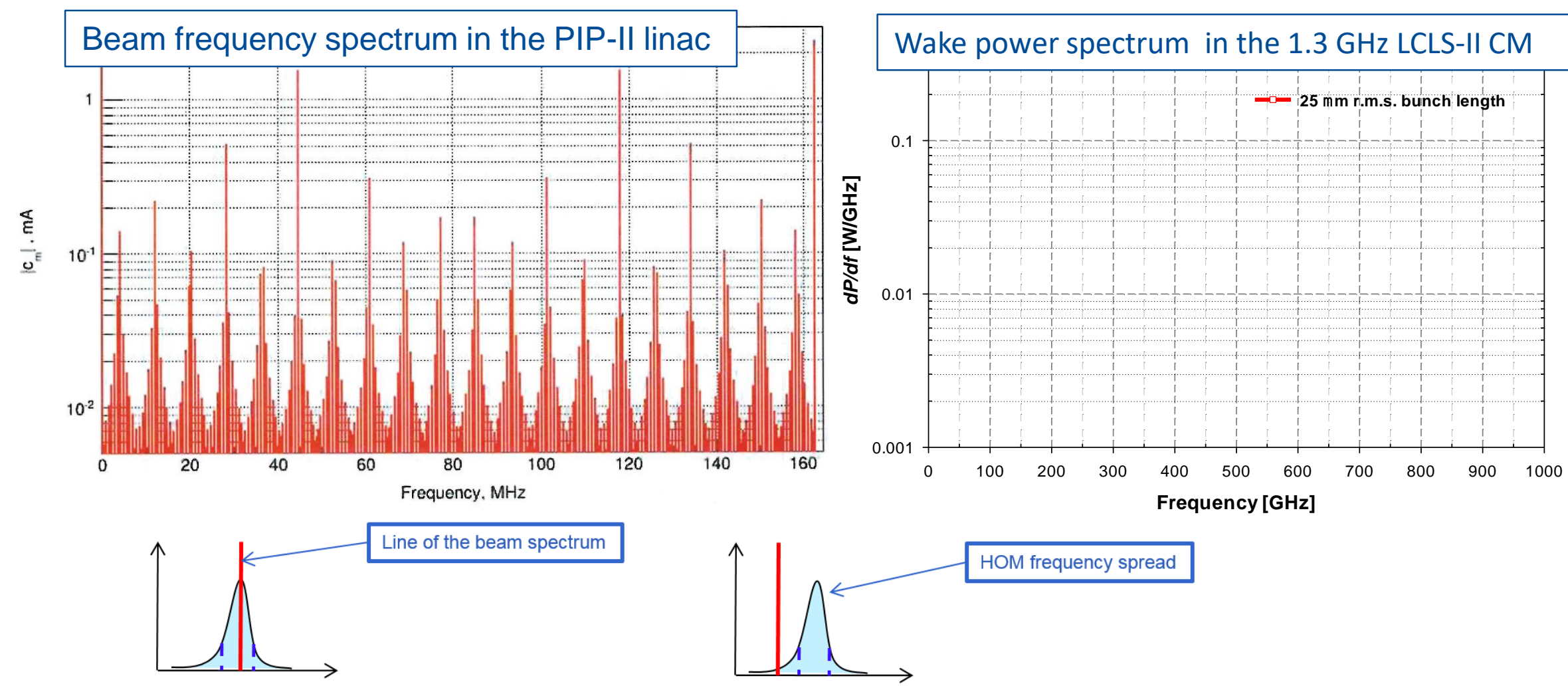

$\langle P\rangle_{\text {max }}=\frac{\left(R_{\|} / Q\right) \omega_{0} q_{0}^{2}}{4 t_{b}}\left(\frac{e^{\alpha}+1}{e^{\alpha}-1}\right):\left\{\begin{array}{l}\alpha=t_{b} / \quad t_{b} \text { is the bunch spacing } \\ \tau=2 Q_{L} \omega_{0} \text { is the HOM signal decay time }\end{array}\right.$

- High bunches rep. rate \& peak beam current might result in large cryogenic losses and beam emittance dilution 


\section{Random Cavity Generation}
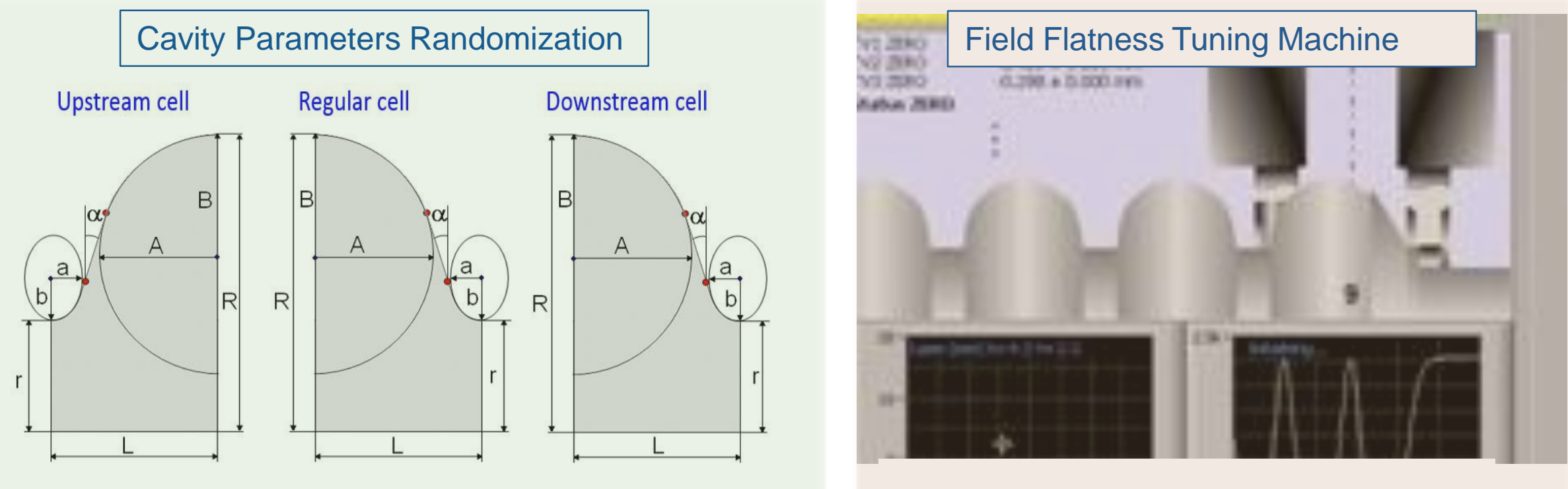

$$
P_{n}^{i}=P_{n}^{n o m}+\left|\Delta_{t o l}\right|[2 R n d(1)-1]
$$

$$
\Delta L^{i} \frac{\partial f}{\partial L^{i}}=-\sum_{n=1}^{N}\left[\Delta P_{n}^{i} \frac{\partial f}{\partial P_{n}^{i}}\right]
$$

$\Delta_{\text {tol }}$ - cavity mechanical tolerance $(\sim 100 . .250 \mu \mathrm{m})$

$\partial f / \partial L^{i}$ and $\partial f / \partial P_{n}^{i}$ - frequency-dependent sensitivities of the $i^{\text {th }}$ half-cell parameters

- We can randomize cavity parameters and keep the field flatness!

- Assumptions:

a) parameter sensitivities are independent, b) tolerances are uncorrelated 


\section{Eigenmode Analysis Setup}

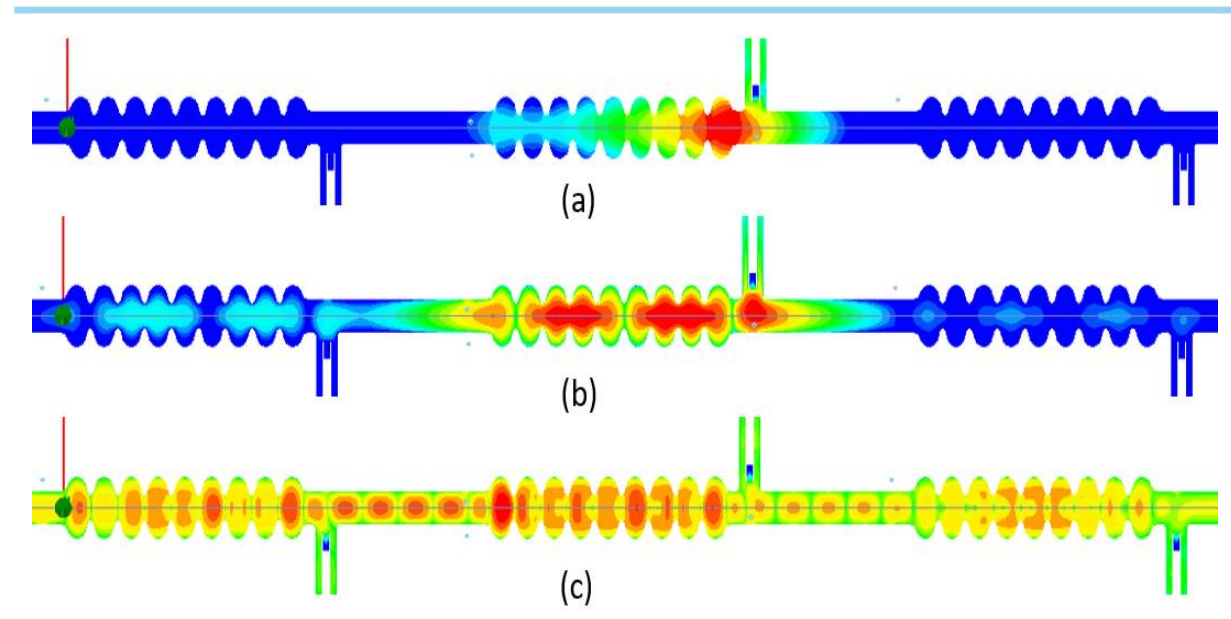

Trapped modes in the infinite chain of random SRF cavities:

a) - High-Q, b) - Medium-Q, c) - Low-Q

- What is a minimum number of SRF cavities is required?

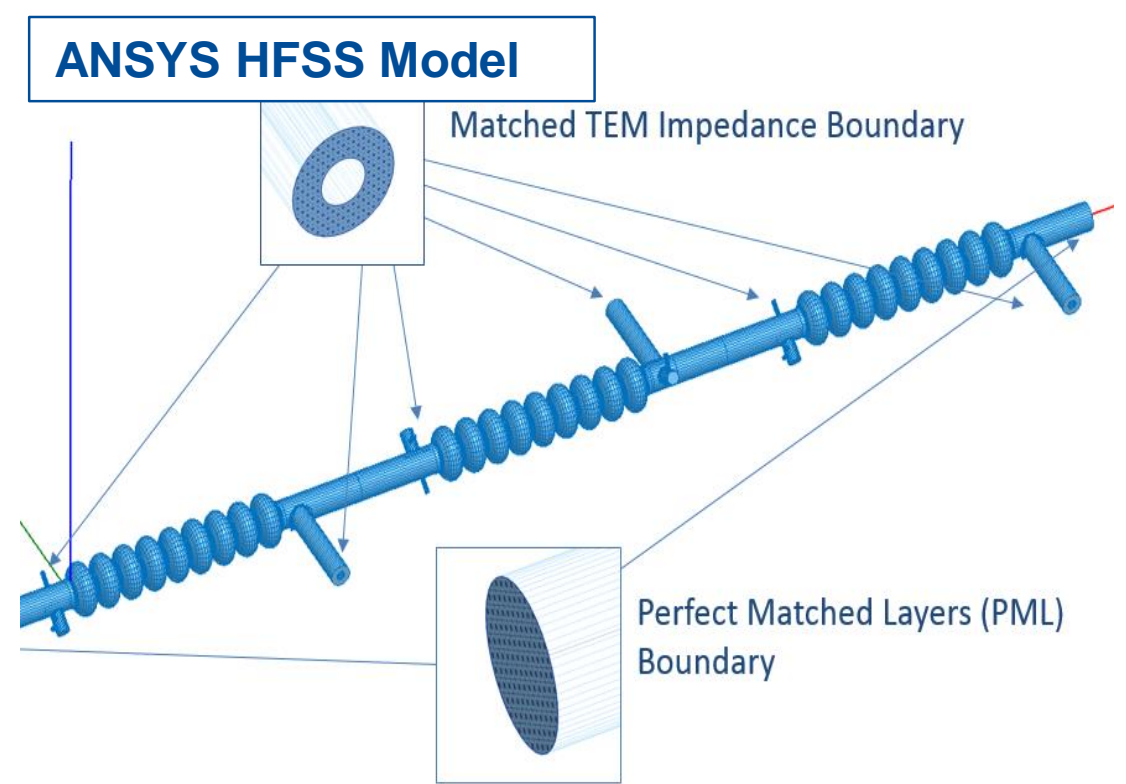

- 1 cavity for HOMs below the beam pipe cut off frequency (TE11, TM01..)

- 3 cavities is the optimum choice for HOMs above the cut off frequency

- >3 cavities give a little or no impact to the overall result.

- Boundary conditions:

- TEM impedance (377 $\Omega$ ) on all coaxial ports

- PML on open beam pipe

- Secondary values (important for the HOMs sorting):

- local stored energy in each cavity and adjacent beam pipes

- longitudinal and transverse R/Q-s

- partial external quality factors for all coupler ports 


\section{Stochastic HOM Analysis (HE 650 MHz PIP-II Cavity*)}

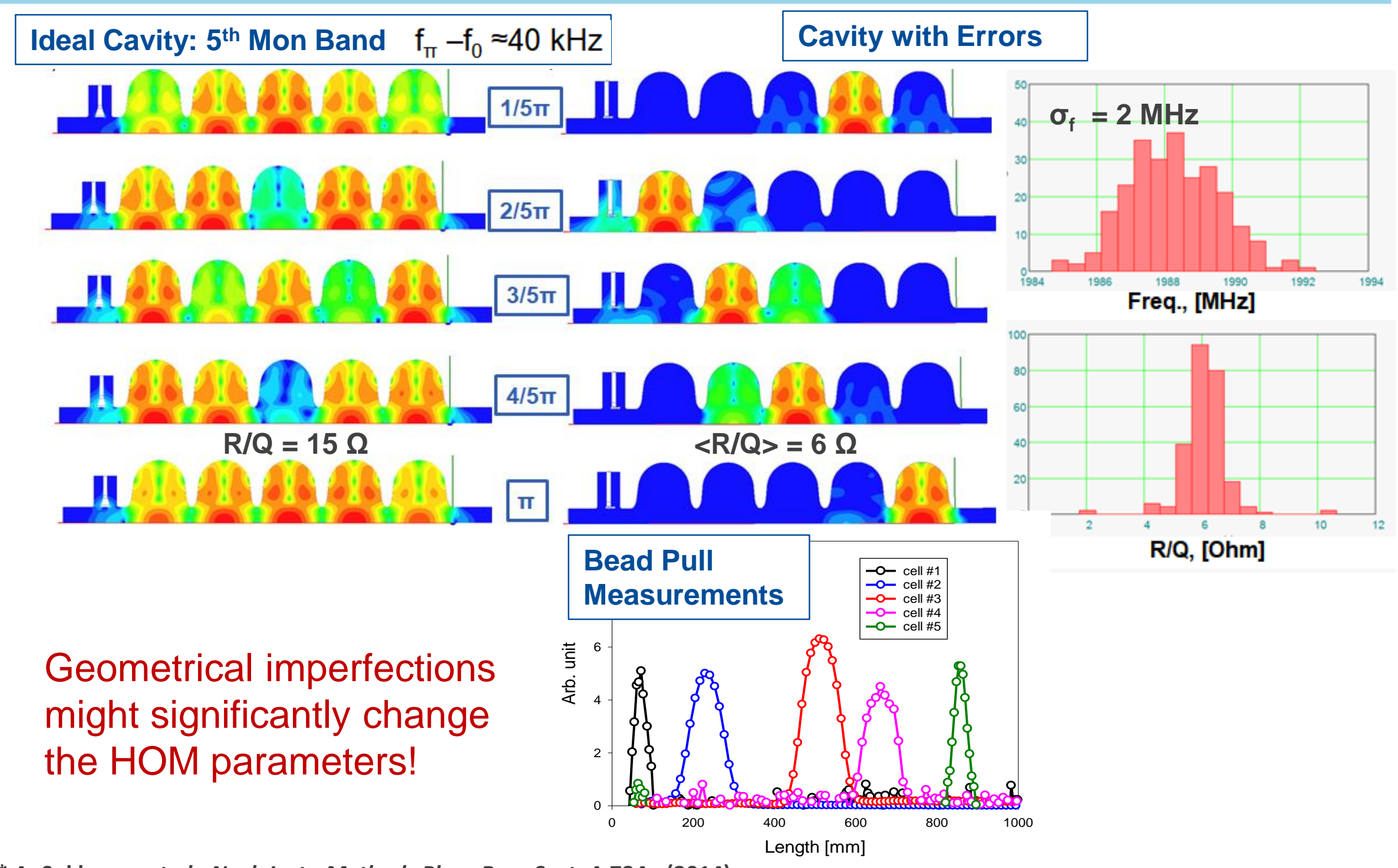

* A. Sukhanov et al., Nucl. Instr. Methods Phys. Res., Sect. A 734,, (2014) 


\section{Stochastic HOM Analysis (3.9 GHz LCLS-II Cavity*)}
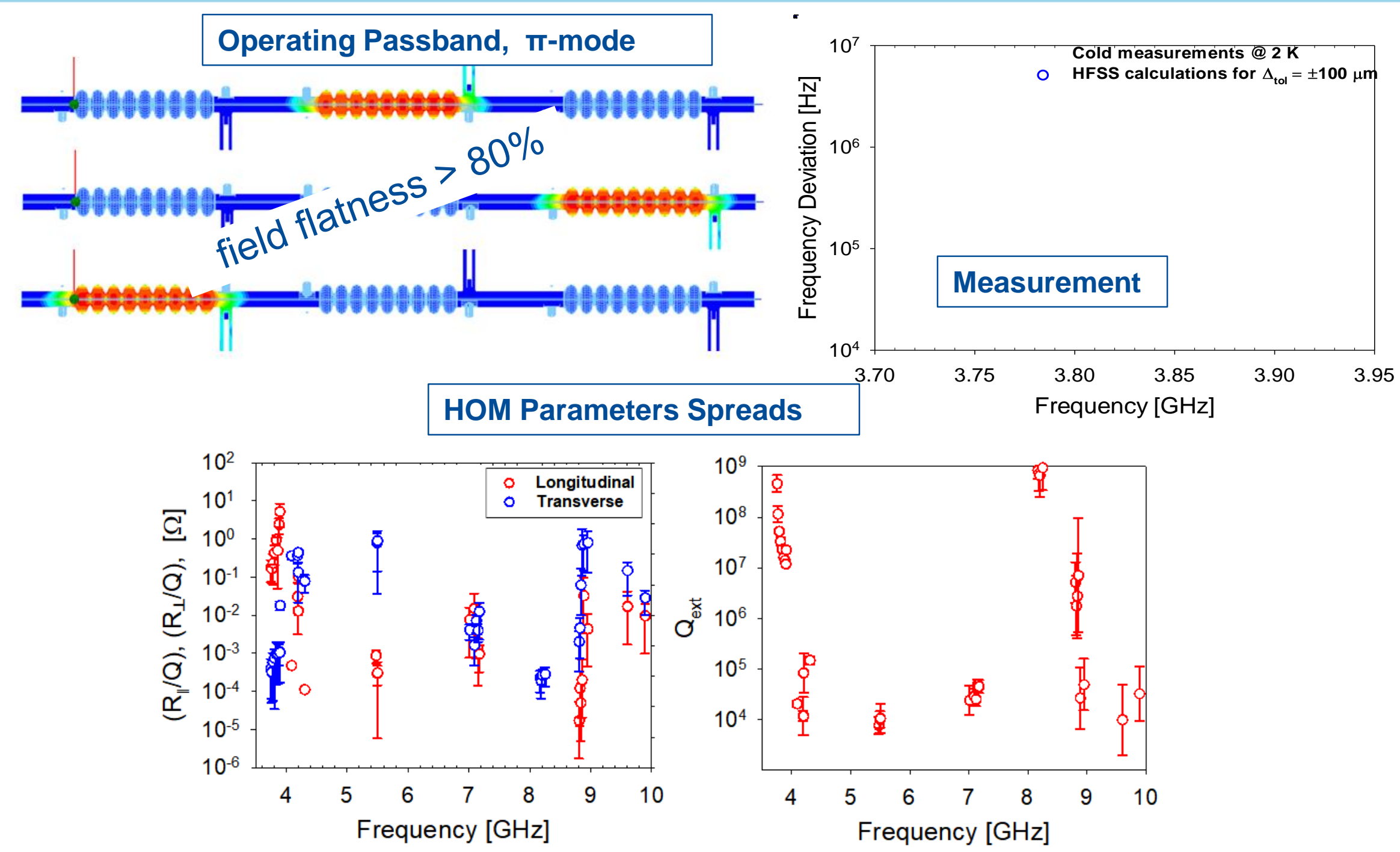

* A. Lunin et al., Phys. Rev. ST AB, 21, 022001 (2018) 


\section{Stochastic HOM Analysis (1.3 GHz LCLS-II Cavity)}
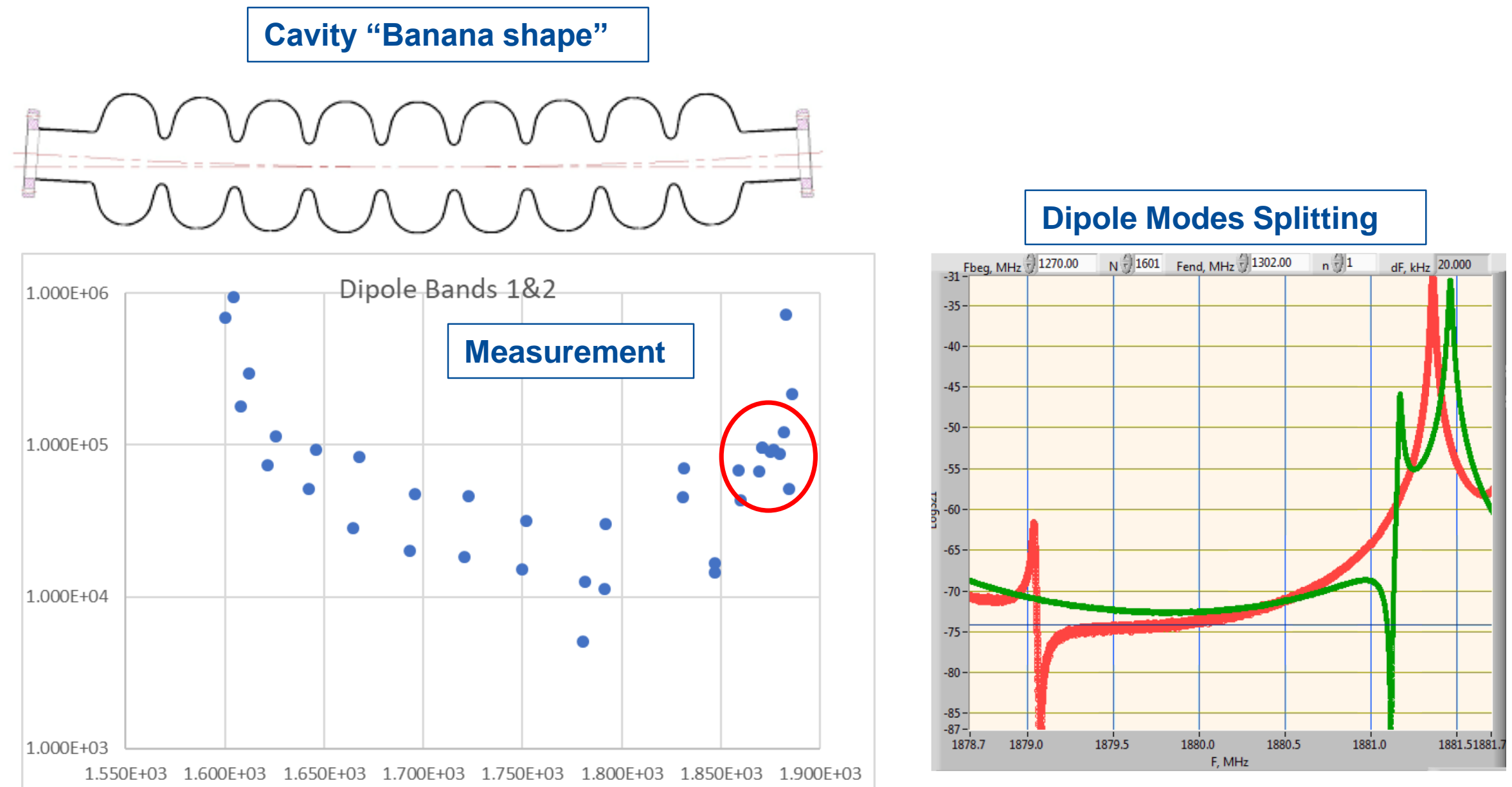

Geometrical imperfections might significantly change the HOM parameters! 


\section{Resonant HOMs Excitation of the $650 \mathrm{MHz}$ PIP-II cavity}

- Statistical approach of resonant HOMs excitation:

- sort out the middle cavity HOMs compendium

- find means and spreads of $F, R / Q, Q$ for each mode

- generate $10^{\mathrm{N}}$ cavities/cryomodules with random HOMs spectra

- calculate probabilities of RF losses and emittance dilution
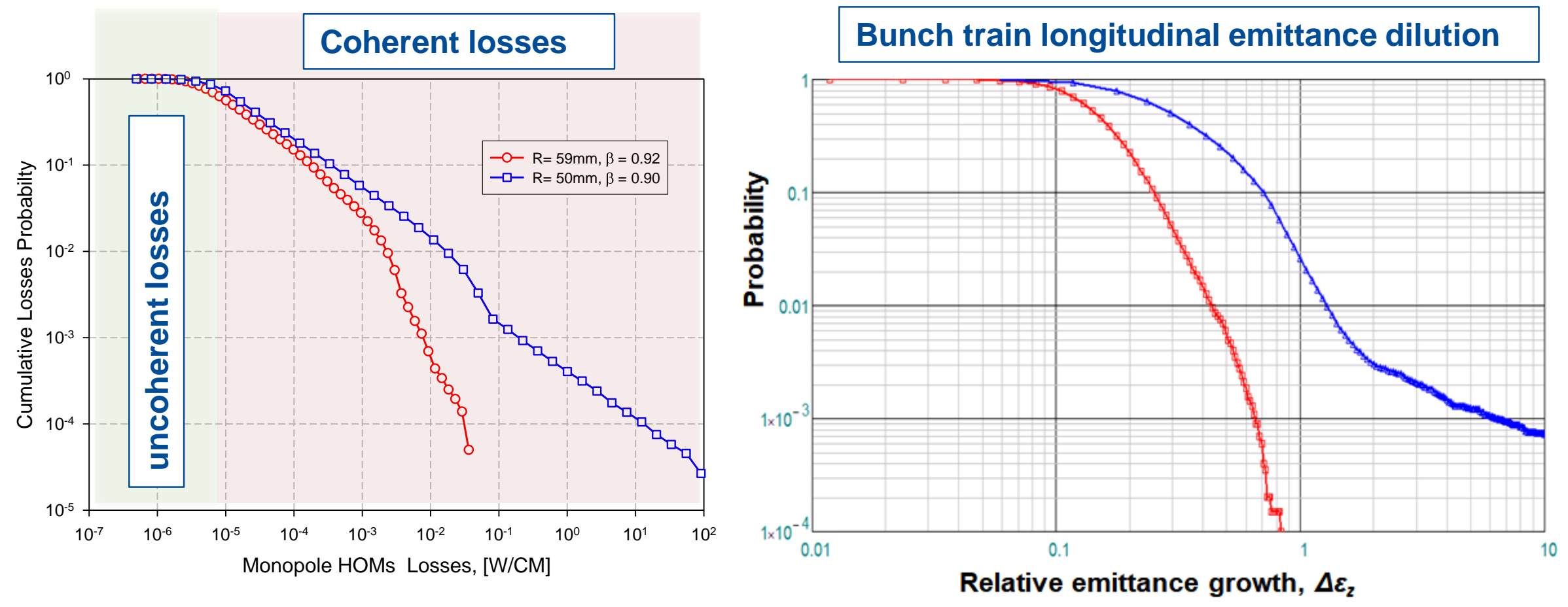

Comparison of two versions (beta 0.90 and 0.92 ) of HE 650 cavity for the PIP-II linac 


\section{Resonant HOMs Excitation of the 3.9 GHz LCLS-II cavity}

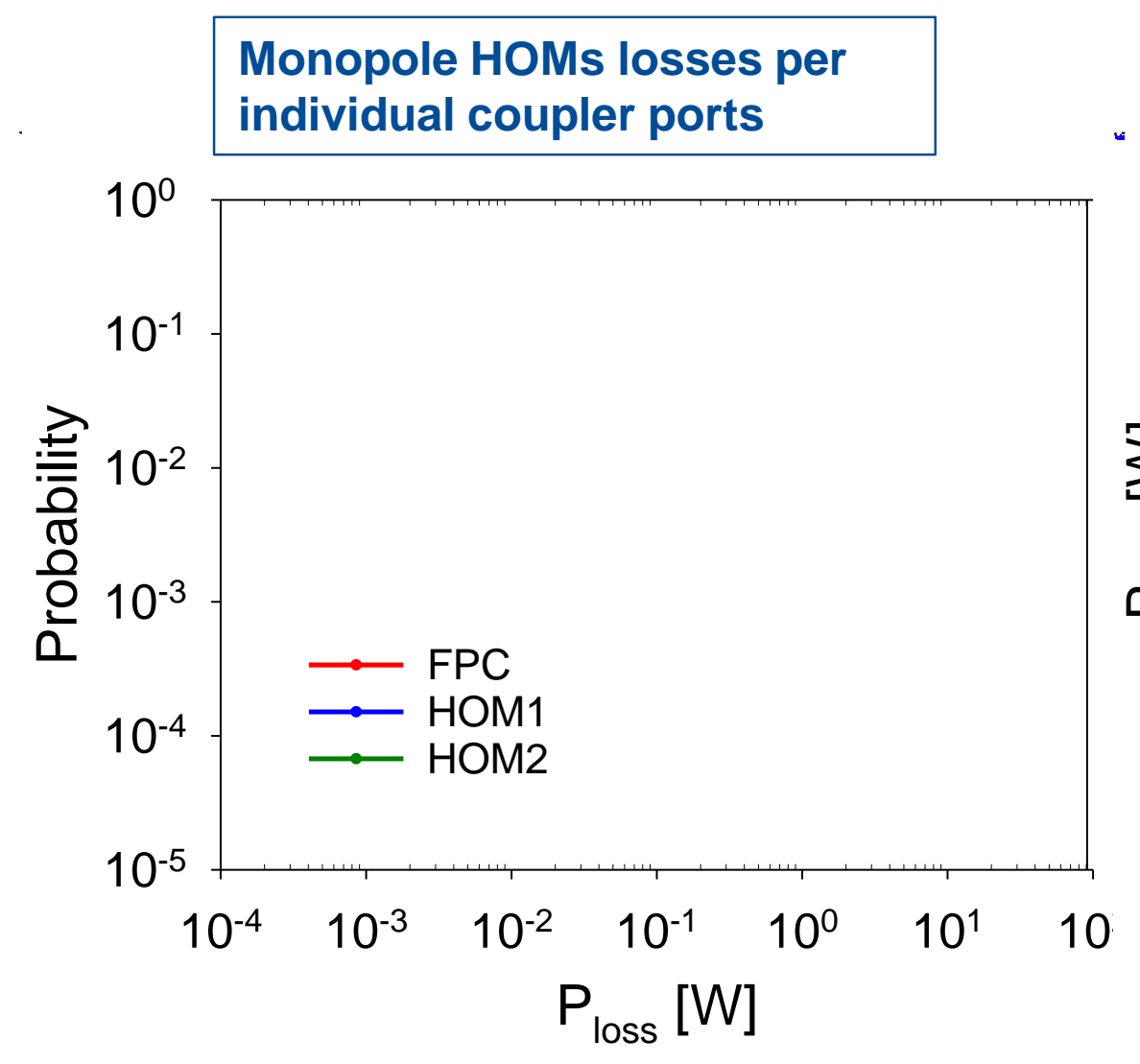

Comparison of XFEL and LSLS-II cavities

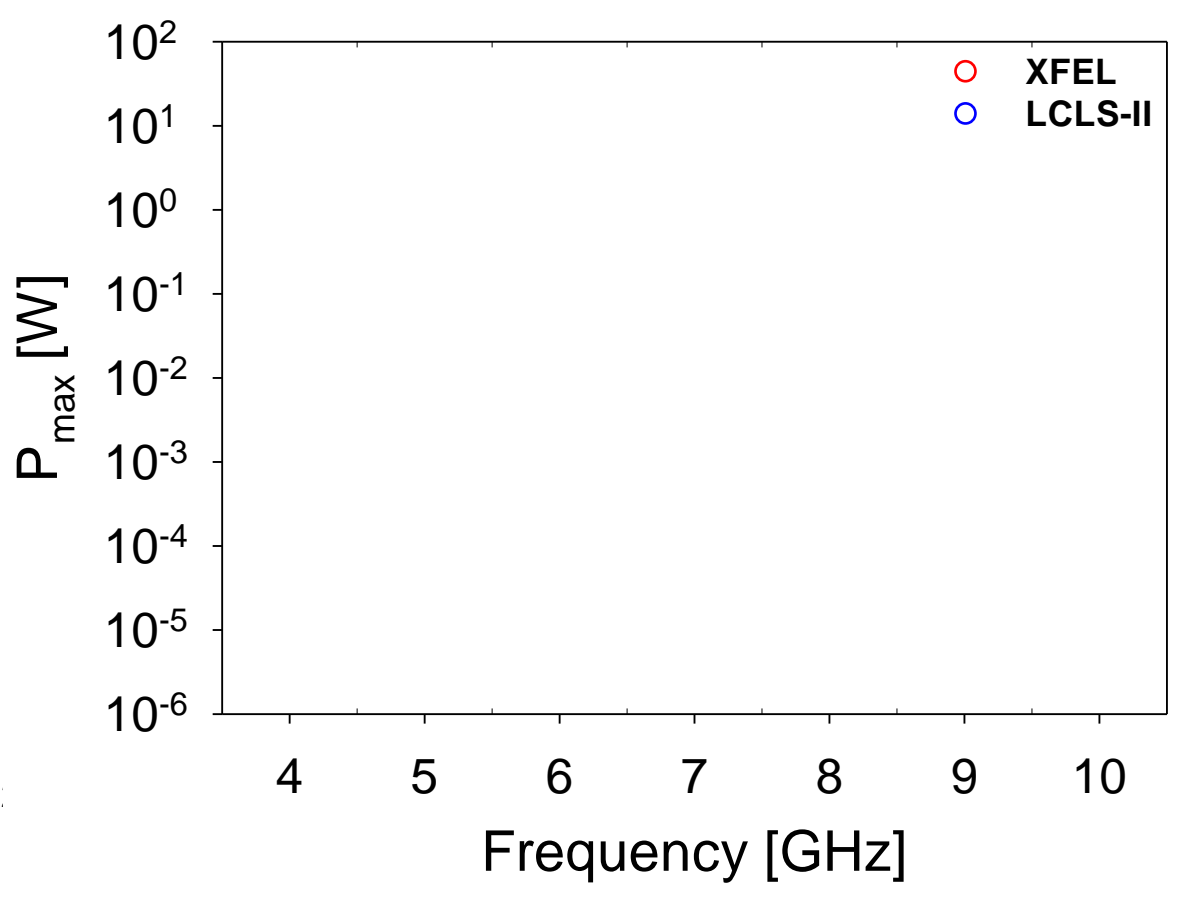

- Modified 3.9 GHz cavity is capable of efficiently damping the resonant excitation of HOMs spectrum by the continuous beam in the LCLS-II linac 


\section{Conclusions}

- The statistical analysis of the eigenmode spectrum in SRF cavities is reliable tool for quantitative evaluation of the coherent HOM excitation by the beam with arbitrary time structure

- The outcome of HOM analysis resulted in critical decisions for the design of superconducting accelerating cavities:

- optimized HE $650 \mathrm{MHz}$ cavity design

- modification of the 3.9 GHz cavity End Group

- Proposed technique can be easily adapted and used for other superconducting particle accelerators operating at high average beam current and high duty factor regimes 